14,13

\title{
Плотность электронных состояний в зоне проводимости сверхтонких пленок дикарбоксильно замещенного и тетракарбоксильно замещенного нафталина на поверхности окисленного кремния
}

\author{
(C) А.С. Комолов ${ }^{1}$, Э.Ф. Лазнева ${ }^{1}$, Н.Б. Герасимова ${ }^{1}$, Ю.А. Панина ${ }^{1}$, \\ А.В. Барамыгин ${ }^{1}$, Г.Д. Зашихин ${ }^{1}$, C.А. Пшеничнюк ${ }^{2}$ \\ ${ }^{1}$ Санкт-Петербургский государственный университет, \\ Санкт-Петербург, Россия \\ ${ }^{2}$ Институт фризики молекул и кристаллов Уфимского научного центра РАН, \\ Уфра, Россия \\ E-mail: a.komolov@spbu.ru
}

(Поступила в Редакцию 27 сентября 2017 г.)

\begin{abstract}
Приведены результаты исследования электронной структуры зоны проводимости пленок дикарбоксильно замещенного нафталина (NDCA) в процессе их осаждения на поверхность окисленного кремния методом спектроскопии полного тока (СПТ) в энергетическом диапазоне от 5 до $20 \mathrm{eV}$ выше уровня Ферми. На основе результатов СПТ экспериментов и теоретических расчетов установлено энергетическое положение основных максимумов плотности незаполненных электронных состояний (DOUS) пленки NDCA и проведено сравнение с положением максимумов DOUS пленки тетракарбоксильно замещенного нафталина (NTCDA). Теоретический анализ включал в себя расчет энергий и пространственного распределения орбиталей исследованных молекул методом теории функционала плотности (DFT) на уровне B3LYP/6-31G(d) и последующую корректировку рассчитанных значений энергий орбиталей по методу, зарекомендовавшему себя ранее при исследованиях зоны проводимости пленок малых сопряженных органических молекул. В исследованном диапазоне энергий от 5 до $20 \mathrm{eV}$ выше уровня Ферми максимумы DOUS пленки NTCDA сдвинуты в сторону меньших энергий электрона на $1-2 \mathrm{eV}$ относительно соответствующих максимумов DOUS пленки NDCA.
\end{abstract}

Работа выполнена при поддержке грантов РФФИ (15-29-05786, 18-03-00020). В работе использовали оборудование научного парка СПбГУ „Методы анализа состава вещества“ и „Диагностика функциональных материалов для медицины, фармокологии и наноэлектроники“.

Авторы благодарят ООО „ЭФА медика“ и генерального директора Артемова В.В. за предоставление ксенонового осветительного оборудования.

DOI: 10.21883/FTT.2018.04.45696.281

\section{1. Введение}

Формирование поверхностных структур на основе сопряженных органических материалов и явления переноса электронного заряда в таких материалах предоставляет широкие возможности для устройств органической электроники [1-4]. В качестве возможных вариантов оптимизации приборных характеристик органических электронных устройств рассматривают введение в состав молекул электрически активных замещающих групп и воздействие на сверхтонкий органический слой со стороны поверхности твердотельной подложки [5-7]. Это представляет значительный фундаментальный интерес в плане установления закономерностей формирования энергетических зон электронных состояний в органических полупроводниках и в пограничной области в гибридных и поверхностных структурах [8-11]. Поверхности оксидов металлов, диоксида кремния, оксида графена рассматривают как перспективные для нанесе- ния сплошных и наноструктурированных сопряженных органических покрытий [11-16]. Достаточно перспективными считают тонкие и сверхтонкие покрытия на основе молекул замещенных периленов и нафталинов, такие как перилен-тетракарбоновый диангидрид (PTCDA) и нафталин-тетракарбоновый диангидрид (NTCDA) [6,17-20]. В результате исследований методами спектроскопии оптического поглощения и методами электронной спектроскопии было показано, что введение в молекулы перилена и нафталина электрон-акцепторных заместителей, содержащих диангидрид или имид карбоновой кислоты, приводит к понижению положения энергетических уровней, соответствующих энергетической зоне проводимости в этих материалах [21,22].

Одним из экспериментальных подходов к изучению плотности незаполненных электронных состояний (DOUS) является методика спектроскопии полного тока (CПТ), дающая возможность исследования энергетических состояний в зоне проводимости посредством тести- 
рующего пучка медленных электронов [23]. Одновременный теоретический анализ с использованием результатов ab initio расчетов позволяет установить природу обнаруженных максимумов DOUS. Ранее мы проводили исследование DOUS пленок PTCDA и NTCDA с помощью СПТ-методики и методики спектроскопии электронного захвата, и было показано, что при толщине пленки более $6 \mathrm{~nm}$ DOUS практически не зависит от материала подложки и достаточно хорошо соответствует структуре вакантных орбиталей изолированных молекул $[22,24,25]$. В данной статье приведены результаты исследования электронной структуры зоны проводимости в процессе формирования интерфейса сверхтонких пленок дикарбоксильно замещенного нафталина (NDCA), молекул, отличающихся от NTCDA меньшим количеством замещающих функциональных групп (рис. 1). Приведено сравнение результатов исследования плотности незаполненных электронных состояний пленок NTCDA и NDCA в диапазоне от 5 до $20 \mathrm{eV}$ выше уровня Ферми $\left(E_{\mathrm{F}}\right)$, полученной на основе экспериментальных исследований методом СПТ и на основе результатов теоретических расчетов с использованием методов теории функционала плотности (DFT).

\section{2. Эксперимент}

Для приготовления исследованных пленок использовали реактивы нафталин-1,4,5,8-тетракарбоновый диангидрид (NTCDA) и нафталин-1,8-дикарбоновый ангидрид (NDCA) (рис. 1), доступные коммерчески (SigmaAldrich). Обезгаживание реактивов проводили путем их выдержки в течение нескольких часов в вакуумной камере при базовом давлении $10^{-6} \mathrm{~Pa}$ и температуре $100^{\circ} \mathrm{C}$. В качестве подложек для нанесения пленок использовали пластины $\left(\mathrm{SiO}_{2}\right) n$ - $\mathrm{Si}$, предварительно очищенные в $10 \%$ растворе $\mathrm{HF}$ и смеси $\mathrm{H}_{2} \mathrm{O}_{2} / \mathrm{H}_{2} \mathrm{SO}_{4}$. После такой процедуры очистки на поверхности кремния формируется слой оксида кремния толщиной около $3-5 \mathrm{~nm}$ с составом, близким к стехиометрическому $[7,12]$. Для формирования пленок проводили термическое осаждение в вакууме органического материала, NDCA или NTCDA, на поверхность подложки со скоростью примерно $0.1 \mathrm{~nm} / \mathrm{min}$. Непосредственно в процессе увеличения толщины органического покрытия до 6-7 nm проводили измерения методом СПТ [23]. Во время осаждения пленок допускали повышение давления на порядок от базового давления в вакуумной камере, при этом не производилось дополнительного нагрева подложки или отжига осажденного органического слоя. Как было показано для ряда различных пленок замещенных нафталинов и периленов, такая процедура осаждения приводит к формированию неупорядоченного органического слоя [25-27]. В методике СПТ параллельный пучок электронов площадью поперечного сечения $0.2-0.4 \mathrm{~mm}$ направляется по нормали к исследуемой поверхности
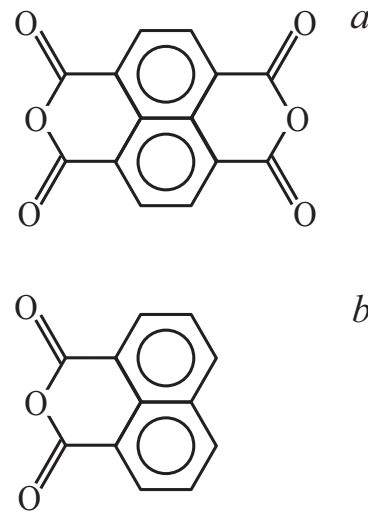

$b$

Рис. 1. Структурные формулы молекул нафталин-1,4,5,8-тетракарбонового диангидрида (NTCDA) (a) и нафталин-1,8-дикарбонового ангидрида (NDCA) $(b)$.

и регистрируется производная по энергии от полного тока $S(E)$, проходящего через образец, как функция энергии падающих электронов, которую варьируют в диапазоне от 0 до $25 \mathrm{eV}$ [23]. Зависимость $S(E)$, тонкая структура спектров полного тока (ТССПТ), отражает изменения вероятности входа падающих низкоэнергетических электронов в исследуемый образец, которая связана с зависимостью плотности незаполненных электронных состояний (DOUS) от энергии исследуемой поверхности [23,24,28]. Теоретический анализ DOUS исследованных пленок проводили путем расчетов методом DFT с помощью гибридного функционала B3LYP [29] в базисе 6-31G(d), реализованном в программном пакете Gaussian [30]. На основе схематических представлений вакантных орбиталей, их симметрии и формы, полученные из расчета, определяли их $\pi^{*}$ - и $\sigma^{*}$-характер, так как $\sigma^{*}$-орбиталь симметрична относительно линии связи, а $\pi^{*}$-орбиталь симметрична относительно плоскости, проходящей через линию связи. К рассчитанным значениям энергий орбиталей (virtual orbital energies, VOE) применяли процедуру корректировки, подробно рассмотренную и опробованную для малых сопряженных органических молекул в работах [31-33]. В результате этой процедуры получают скорректированные значения энергий орбиталей (scaled virtual orbital energies, SVOE). В случае $\pi^{*}$-молекулярных орбиталей применяют выражение $\mathrm{SVOE}=(\mathrm{VOE}+1.14) / 1.24$, а в случае $\sigma^{*}$-орбиталей выражение SVOE $=(\mathrm{VOE}+0.97) / 0.79$ [31,32,34].

\section{3. Результаты и обсуждение}

Серия зависимостей тонкой структуры спектров полного тока (ТССПТ), измеренная в процессе осаждения покрытия NDCA на поверхность $\left(\mathrm{SiO}_{2}\right) n-\mathrm{Si}$, представлена на рис. $2, b$. ТССПТ, измеренная до начала осаждения NDCA-пленки, соответствует обозначению „0 $0 \mathrm{~nm}^{\text {“ }}$ толщины органического покрытия (рис. $2, b$ ), исходит 


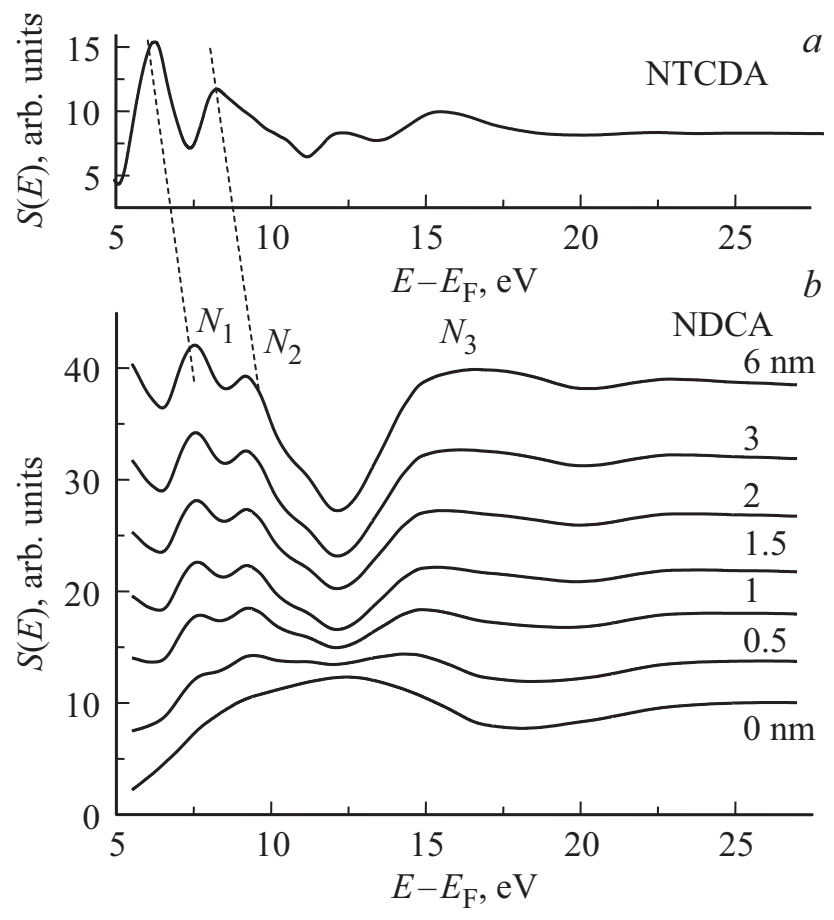

Рис. 2. $a-$ TCCПT пленки NTCDA толщиной $6 \mathrm{~nm}$ на поверхности $\left(\mathrm{SiO}_{2}\right) n-\mathrm{Si} . \quad b-$ ТССПТ в процессе осаждения пленки NDCA на поверхность $\left(\mathrm{SiO}_{2}\right) n-\mathrm{Si} . N_{1}-N_{3}-$ максимумы ТССПТ, характерные для слоя NDCA. Подписи около кривых указывают соответствующую толщину пленки NDCA. Пунктирные линии показаны для удобства сравнения положений максимумов.

от подложки и обладает характерной для поверхности $\left(\mathrm{SiO}_{2}\right) n-\mathrm{Si}$ формой, наблюдавшейся ранее для других образцов $\left(\mathrm{SiO}_{2}\right) n-\mathrm{Si}[7,23]$. Так, для ТССПТ поверхности $\left(\mathrm{SiO}_{2}\right) n-\mathrm{Si}$ характерен широкий максимум в области энергий от 6 до $16 \mathrm{eV}$, в котором можно различить по крайней мере два компонента при 9 и $12.5 \mathrm{eV}$. По мере осаждения органического покрытия ТССПТ $\left(\mathrm{SiO}_{2}\right) n$-Si подложки затухала, одновременно нарастала интенсивность новой ТCCПT, отражающей электронную структуру зоны проводимости NDCA покрытия (рис. 2,b). Для ТССПТ NDCA-пленки характерны максимумы $N_{1}, N_{2}$, расположенные при энергиях $7.5,9.0 \mathrm{eV}$, и достаточно широкий максимум $N_{3}$ с двумя выраженными компонентами при 15.0 и $17.5 \mathrm{eV}$ (рис. $2, b$ ). Для анализа характера изменения интенсивности ТССПТ осаждаемого покрытия удобно выбрать максимум $N_{2}$ при энергии $9.0 \mathrm{eV}$. Для электронов с энергией $9 \mathrm{eV}$ выше уровня $E_{\mathrm{F}}$ длина свободного пробега $(\lambda)$ составляет приблизительно $4 \mathrm{~nm}[23,35]$. Для оценки изменений интенсивности ТССПТ сигнала, исходящего от $\left(\mathrm{SiO}_{2}\right) n$-Si подложки, удобно выбрать разницу между высотой максимума на $12.5 \mathrm{eV}$ относительно минимума, расположенного на $17.5 \mathrm{eV}$ (рис. 2,b). При этих значениях энергии электрона $\lambda$ составляет приблизительно $3 \mathrm{~nm}$. Было обнаружено, что в зависимости от толщины осаждаемого покрытия NDCA интенсивность максимумов подложки экспоненциально убывает, а интенсивность максимума $N_{2}$ растет экспоненциально, что свидетельствует о формировании сплошного напыляемого органического покрытия NDCA, как подробно обсуждалось в работах $[7,23]$. По достижении NDCA-пленки толщины $6 \mathrm{~nm}$ сформировалась стабильная ТССПТ с характерными максимумами $N_{1}, N_{2}$ и $N_{3}$, которая практически не изменялась при увеличении толщины органического покрытия до $8-11 \mathrm{~nm}$. Дальнейшее осаждение органического покрытия приводило к искажениям в ТССПТ вследствие зарядки поверхности под действием падающего электронного пучка.

ТССПТ, отражающая электронную структуру зоны проводимости пленок NTCDA, при толщине пленки примерно $6 \mathrm{~nm}$ на $\left(\mathrm{SiO}_{2}\right) n$-Si подложке представлена на рис. 2,a. Как нами было показано ранее при исследованиях NTCDA покрытий, эта ТCCПT соответствует сформировавшейся пленке и не зависит от материала выбранной подложки [22,24]. Это хорошо соответствует и результатам исследований интерфейсов при формировании пленок NTCDA и пленок на основе других малых сопряженных органических молекул с поверхностями металлов и полупроводников, согласно которым влияние поверхности подложки в большинстве случаев ограничено тонким пограничным слоем толщиной до $2 \mathrm{~nm}[2,12,17,19]$. ТССПТ пленки NTCDA представлена четырьмя основными максимумами с вершинами при $6.5,8.0,12.5$ и $15.5 \mathrm{eV}$. Первые два максимума отражают границы энергетических зон $\pi^{*}$-состояний, согласно результатам теоретических расчетов и исследований методом обращенной фотоэмиссионной спектроскопии $[19,36]$. Максимумы при энергиях выше $12 \mathrm{eV}$ над $E_{\mathrm{F}}$ обладают преимущественно $\sigma^{*}$ - характером, согласно результатам работ $[18,22]$. В результате сравнения TCCПT NTCDA и NDCA пленок авторы делают предположение, что максимумы $N_{1}$ и $N_{2}$ TCCПТ пленки NDCA являются аналогами $\pi^{*}$-максимумов пленки NTCDA и сдвинуты в сторону бо́льших значений энергий электрона примерно на $1 \mathrm{eV}$ (рис. 2, см. также пунктирные линии). Максимум $N_{3}$ TCCПТ пленки NDCA, вероятно, имеет $\sigma^{*}$-природу, аналогично максимумам при энергиях 12.5 и $15.5 \mathrm{eV}$.

Экспериментальные ТССПТ можно использовать в качестве основы для построения зависимостей плотности незаполненных электронных состояний в зоне проводимости (DOUS). Как было показано в ряде работ, структура максимумов DOUS соответствует структуре максимумов ТССПТ и может быть построена в виде производной зависимости $S(E)$ с обратным знаком: $-d S(E) / d E[24,28,34]$. В случае исследованных пленок NTCDA и NDCA для построения экспериментальной DOUS использовали зависимости ТССПТ, представленные на рис. 2. Таким образом, было установлено, что DOUS пленок NTCDA в энергетическом диапазоне от 5 до $20 \mathrm{eV}$ выше $E_{\mathrm{F}}$ представлена максимумами 

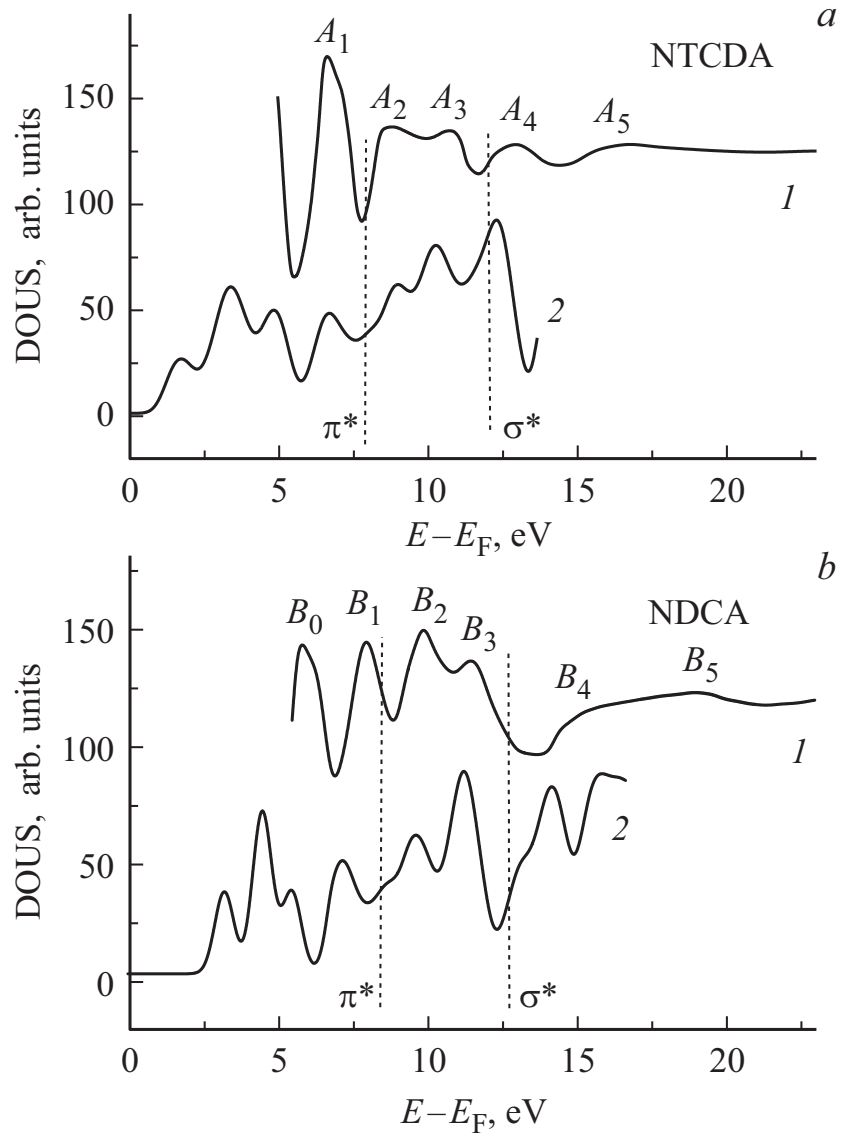

Рис. 3. DOUS исследованных сопряженных органических пленок. $a-$ пленок NTCDA и $b-$ пленок NDCA. Кривые $1-$ DOUS зависимости, построенные на основе экспериментальных ТСCПТ. Кривые 2 - DOUS зависимости, построенные на основе результатов расчетов с использованием методов DFT на уровне B3LYP/6-31G(d). $A_{1}-A_{5}$ - обозначения основных максимумов DOUS пленок NTCDA, $B_{0}-B_{5}$ - обозначения основных максимумов DOUS пленок NDCA. Вертикальные пунктирные линии разделяют области преимущественного обнаружения максимумов DOUS $\pi^{*}$ - и $\sigma^{*}$-типа и переходную область, где максимумы DOUS имеют вклады и от $\pi^{*}$-, и от $\sigma^{*}$-электронных орбиталей.

$A_{1}-A_{5}$, a DOUS пленок NDCA в этом энергетическом диапазоне представлена максимумами $B_{0}-B_{5}$ (рис. 3, кривые 1). Для анализа экспериментальных зависимостей DOUS проведен расчет энергий орбиталей исследованных молекул NTCDA и NDCA методом на уровне $\mathrm{B} 3 \mathrm{LYP} / 6-31 \mathrm{G}(\mathrm{d})$ и последующая корректировка [31-33], как обсуждалось подробнее в разд. 2. В результате операции свертки рассчитанных энергий орбиталей с функцией Гаусса, полушириной $0.5 \mathrm{eV}$ построены зависимости DOUS (рис. 3, кривые 2). При сравнении теоретических и экспериментальных DOUS зависимостей достаточно хорошее соответствие энергетического расположения максимумов наблюдается в диапазоне от 6 до $12 \mathrm{eV}$ выше $E_{\mathrm{F}}$ (рис. 3). Так, экспериментальным DOUS максимумам $A_{1}-A_{3}$ в случае пленок NTCDA и экспериментальным DOUS максимумам $B_{0}-B_{3}$ в случае пленок NDCA (рис. 3, кривые 1) соответствуют максимумы на теоретически рассчитанных DOUS зависимостях (рис. 3, кривые 2). Значения энергий ниже 5-6 eV над $E_{\mathrm{F}}$ выходят за рамки диапазона, в котором DOUS может быть установлена с использованием результатов СПТ эксперимента. При энергии выше $12 \mathrm{eV}$ над $E_{\mathrm{F}}$ экспериментальные DOUS демонстрируют достаточно широкие максимумы $A_{4}, A_{5}$ в случае пленок NTCDA и максимумы $B_{4}, B_{5}$ в случае пленок NDCA (рис. 3). Вместе с этим расчетные зависимости DOUS имели при энергиях выше $12 \mathrm{eV}$ над $E_{\mathrm{F}}$ отчетливую структуру, состоящую из более узких максимумов. Это различие следует связывать с тем, что при увеличении энергии падающего электрона более $12 \mathrm{eV}$ выше $E_{\mathrm{F}}$ происходит существенное размытие экспериментальной ТССПТ.

Результаты проведенного DFT-расчета позволили также визуализировать форму, симметрию и локализацию вакантных орбиталей молекул NTCDA и NDCA с помощью используемого программного пакета [30]. Это позволило установить $\pi^{*}$ - или $\sigma^{*}$-характер максимумов в рассчитанных DOUS зависимостях. Для обеих пленок, NTCDA и NDCA, установлено, что при энергиях менее $8.0 \mathrm{eV}$ над $E_{\mathrm{F}}$ расположены преимущественно $\pi^{*}$-орбитали, а в диапазоне энергий от 8.0 до $12.5 \mathrm{eV}$ располагается переходная область, в которой присутствуют и $\pi^{*}$ - и $\sigma^{*}$-орбитали. Таким образом, максимумы DOUS $A_{1}, B_{0}$ и $B_{1}$ (рис. 3) сформированы $\pi^{*}$-орбиталями, максимумы $A_{2}, A_{3}, B_{2}$ и $B_{3}$ имеют смешанную, $\pi^{*}$ и $\sigma^{*}$, природу. Более высоколежащие максимумы $A_{4}, A_{5}, B_{4}$ и $B_{5}$ (рис. 3) имеют преимущественно $\sigma^{*}$-характер. О наличии максимумов DOUS, имеющих смешанную, $\pi^{*}$ и $\sigma^{*}$, природу, сообщалось в литературе ранее [37,38]. Так, при замещении молекул бензола электрон-притягивающими функциональными группами было обнаружено, что происходил энергетический сдвиг части низколежащих $\sigma^{*}$-орбиталей в область, характерную для расположения DOUS максимумов $\pi^{*}$-типа [37]. Смешанные, $\pi^{*}$ и $\sigma^{*}$, энергетические области DOUS при энергиях около $10 \mathrm{eV}$ выше $E_{\mathrm{F}}$, шириной в несколько $\mathrm{eV}$ были также обнаружены нами ранее в результате исследований пленок замещенных фуллеренов и ряда производных периленов $[27,34]$.

Рассмотрим влияние введения дополнительной замещающей группы, дикарбоксильного диангидрида, в молекуле NDCA и формирование, таким образом, молекулы NTCDA (рис. 1) путем сравнения зависимостей DOUS (рис. 3) пленок на основе этих молекул. В области энергий выше $12.5 \mathrm{eV} \quad \sigma^{*}$-максимумы $A_{4}, A_{5}$ пленки NTCDA сдвинуты на $1.5-2 \mathrm{eV}$ в сторону меньших энергий электрона относительно $\sigma^{*}$-максимумов $B_{4}, B_{5}$ пленки NDCA. В диапазоне энергий от 6 до $12.5 \mathrm{eV}$ максимумы $A_{1}-A_{3}$ пленки NTCDA также сдвинуты в сторону меньших энергий электрона до $1 \mathrm{eV}$ относительно соответствующих максимумов $B_{1}-B_{3}$ пленки NDCA. 
Максимуму $B_{0}$ при энергии $5.5 \mathrm{eV}$ пленки NDCA, видимо, соответствует максимум пленки NTCDA, расположенный при энергии $4.8 \mathrm{eV}$, однако в случае NTCDA этот максимум удалось зарегистрировать только в расчетной зависимости DOUS (рис. 3, $a$, кривая 2). Относительный энергетический сдвиг расположения $\pi^{*}$ - и низколежащих $\sigma^{*}$-максимумов наблюдался для случаев NTCDA и NDCA ранее в результатах исследований методом спектроскопии электронного захвата [24], это различие следует связывать с изменением области сопряжения в области нафталинового остова исследованных молекул [39].

\section{4. Заключение}

Исследованы закономерности формирования электронной структуры зоны проводимости пленок дикарбоксильно замещенного нафталина (NDCA) на поверхности окисленного кремния в энергетическом диапазоне от 5 до $20 \mathrm{eV}$ над уровнем Ферми при использовании методики низкоэнергетической электронной СПТ и установлена структура максимумов ТССПТ пленок NDCA. На основе результатов СПТ экспериментов и теоретических расчетов на уровне B3LYP/6-31G(d) с дополнительной корректировкой установлено энергетическое положение основных максимумов DOUS пленки NDCA и проведено сравнение с положением максимумов DOUS пленки NTCDA. Для обеих пленок, NTCDA и NDCA, установлено, что при энергиях менее $8.0 \mathrm{eV}$ над $E_{\mathrm{F}}$ расположены преимущественно $\pi^{*}$-орбитали, а в диапазоне энергий от 8.0 до $12.5 \mathrm{eV}$ располагается переходная область, в которой присутствуют $\pi^{*}$ - и $\sigma^{*}$-орбитали. В исследованном диапазоне энергий от 5 до $20 \mathrm{eV}$ относительно $E_{\mathrm{F}}$ максимумы DOUS пленки NTCDA сдвинуты в сторону меньших энергий электрона на $1-2 \mathrm{eV}$ относительно соответствующих максимумов DOUS пленки NDCA.

\section{Список литературы}

[1] P.A. Troshin, D.K. Susarova, E.A. Khakina, A.A. Goryachev, O.V. Borshchev, S.A. Ponomarenko, V.F. Razumov, N. Serdar Sariciftci. J. Mater. Chem. 22, 18433 (2012).

[2] M. Gruenewald, L.K. Schirra, P. Winget, M. Kozlik, P.F. Ndione, A.K. Sigdel, J.J. Berry, R. Forker, J.-L. Brédas, T. Fritz, O.L.A. Monti. J. Phys. Chem. C 119, 4865 (2015).

[3] A.N. Aleshin, I.P. Shcherbakov, A.S. Komolov, V.N. Petrov, I.N. Trapeznikova. Organic Electron. 16, 186 (2015).

[4] А.С. Комолов, Э.Ф. Лазнева, Н.Б. Герасимова, Ю.А. Панина, А.В. Барамыгин, Г.Д. Зашихин. ФТТ 58, 1216 (2016).

[5] J.L. Bredas, A.J. Heeger. Chem. Phys. Lett. 217, 507 (1994).

[6] R. Tonner, P. Rosenowa, P. Jakob. Phys. Chem. Chem. Phys. 18, 6316 (2016).
[7] A.S. Komolov, E.F. Lazneva, S.N. Akhremtchik. App. Surf. Sci. 256, 2419 (2010).

[8] I.S. Yahia, H.Y. Zahran, F.H. Alamri. Synth. Met. 222, 186 (2016).

[9] C. Farley, N.V.S.D.K. Bhupathiraju, B.K. John, C.M. Drain. J. Phys. Chem. A 120, 7451 (2016).

[10] B. Handke, L. Klita, J. Niziol, W. Jastrzebski, A. Adamczyk. J. Molec. Struct. 1065-1066, 248 (2014).

[11] B. Handke, L. Klita, W. Niemiec. Surf. Sci. (2017). DOI: 10.1016/j.susc.2017.08.23

[12] L. Grzadziel, M. Krzywiecki, H. Peisert, T.Chassé, J. Szuber. Organic Electron. 13, 1873 (2012).

[13] И.А. Аверин, А.А. Карманов, В.А. Мошников, И.А. Пронин, С.Е. Игошина, А.П. Сигаев, Е.И. Теруков. ФТТ 12, 2304 (2015).

[14] M. Krzywiecki, L. Grzadziel, A. Sarfraz, D. Iqbal, A. Szwajca, A. Erbe. Phys. Chem. Chem. Phys. 17, 10004 (2015).

[15] I.B. Olenych, O.I. Aksimentyeva, L.S. Monastyrskii, Y.Y. Horbenko, M.V. Partyka, A.P. Luchechko, L.I. Yarytska. Nanoscale Res. Lett. 11, 43 (2016).

[16] A.S. Komolov, Y.M. Zhukov, E.F. Lazneva, A.N. Aleshin, S.A. Pshenichnuk, N.B. Gerasimova, Yu.A. Panina, G.D. Zashikhin, A.V. Baramygin. Mater. Des. 113, 319 (2017).

[17] C.K. Chan, E.G. Kim, J.L. Bredas, A. Kahn. Adv. Funct. Mater. 16, 831 (2006).

[18] J. Taborski, P. Vaterlein, U. Zimmermann, E. Umbach. J. Electron. Spectr. Rel. Phen. 75, 129 (1995).

[19] I.G. Hill, J. Schwartz, A. Kahn. Organic Electron. 1, 5 (2000).

[20] D. Ozdal, N.P. Aydinlik, J.B. Bodapati, H. Icil. Photochem. Photobiol. Sci. 16, 262 (2017).

[21] F. Wurthner, C. Thalacker, S. Diele, C. Tschierske. Chem. Eur. J. 7, 2245 (2001).

[22] A.S. Komolov, P.J. Møller, Y.G. Aliaev, E.F. Lazneva, S. Akhremtchik, F.S. Kamounah, J. Mortenson, K. Schaumburg. J. Molec. Struct. 744/747, 145 (2005).

[23] A.S. Komolov, E.F. Lazneva, S.N. Akhremtchik, N.S. Chepilko, A.A. Gavrikov. J. Phys. Chem. C 117, 24, 12633 (2013).

[24] S.A. Pshenichnyuk, A.S. Komolov. J. Phys. Chem. A 116, 1, 761 (2012).

[25] С.А. Пшеничнюк, А.В. Кухто, И.Н. Кухто, А.С. Комолов. ЖТФ 81, 6, 8 (2011).

[26] S. Heutz, A.J. Ferguson, G. Rumbles, T.S. Jones. Organic Electron. 3, 119 (2002).

[27] А.С. Комолов, Э.Ф. Лазнева, Н.Б. Герасимова, Ю.А. Панина, А.В. Барамыгин, Г.Д. Зашихин, С.А. Пшеничнюк. ФТТ 59, 359 (2017).

[28] I. Bartos. Progr. Surf. Sci. 59, 197 (1998).

[29] A.D. Becke. J. Chem. Phys. 98, 5648 (1993).

[30] M.J. Frisch, G.W. Trucks, H.B. Schlegel, G.E. Scuseria, M.A. Robb, J.R. Cheeseman, G. Scalmani, V. Barone, B. Mennucci, G.A. Petersson et al. Gaussian 09, Revision D.01, Gaussian, Inc., Wallingford CT, 2009.

[31] P.D. Burrow, A. Modelli. SAR and QSAR in Env. Res. 24, 647 (2013).

[32] A.M. Scheer, P.D. Burrow. J. Phys. Chem. B 110, 17751 (2006). 
[33] N.L. Asfandiarov, S.A. Pshenichnyuk, A.S. Vorob'ev, E.P. Nafikova, A. Modelli. Rapid Commun. Mass Spectrom. 29, 910 (2015).

[34] A.S. Komolov, E.F. Lazneva, N.B. Gerasimova, Yu.A. Panina, G.D. Zashikhin, A.V. Baramygin, P. Si, S.N. Akhremtchik, A.A. Gavrikov. J. Electron Spectr. Rel. Phen. 205, 52 (2015).

[35] T. Graber, F. Forster, A. Schoell, F. Reinert. Surf. Sci. 605, 878 (2011).

[36] T. Maruyama, A. Hirasawa, T. Shindow, K. Akimoto, H. Kato, A. Kakizaki. J. Lumin. 87-89, 782 (2000).

[37] A.P. Hitchcock, P. Fischer, A. Gedanken, M.B. Robin. J. Phys. Chem. 91, 531 (1987).

[38] J.G. Chen. Surf. Sci Rep. 30, 1 (1997).

[39] A. Schoell, Y. Zou, D. Huebner, S.G. Urquhart, T. Schmidt, R. Fink, E. Umbach. J. Chem. Phys. 123, 044509 (2005). 